\title{
Organogel formation rationalized by Hansen solubility parameters
}

\author{
Matthieu Raynal, ${ }^{a, b}$ and Laurent Bouteiller*a,b
}

\begin{abstract}
${ }_{5}$ Some organic compounds gelate particular solvents by forming a network of anisotropic fibres. We show that Hansen solubility parameters can be used to predict the range of solvents that are likely to be gelled by any given gelator.
\end{abstract}

Gelation of fluids by a limited amount (typically 1 weight $\%$ 10 or less) of a low molecular weight gelator (LMWG) is possible if the LMWG self-assembles into elongated fibrillar structures, which form an entangled network. ${ }^{1,2}$ In spite of their non-covalent nature, such nanostructures were shown to be strong enough to block the flow of liquids, and even to 15 improve the mechanical properties of solids such as polymers, ${ }^{3}$ or bitumen. ${ }^{4}$ The desire to understand the underlying relationship between molecular structure and gelation, as well as the applied aspects of this phenomenon, has been a great driving force in the study of LMWGs. ${ }^{5-11}$

20 Potentially these materials can be used in numerous applications, such as tissue engineering, sensors, pollutant removal, or as templates for nanoparticles and hybrid materials. In this respect, the design of an entirely new gelator molecule for a particular application (i.e. in a particular 25 solvent) is a difficult task. However, the large body of experimental data accumulated over the years should now allow a different approach. Indeed, if one wants to gelate a common organic solvent, it is straightforward to search the literature and find compounds that have been described to 30 form gels in this particular solvent. Of course, for many applications, the fluid of interest (edible oil, cosmetic formulation, solvent mixture for coatings, ...) may not have been tested. Therefore, one needs guiding principles to predict if a previously described compound will gelate a given fluid, 35 based on its known ability to gelate a range of more or less similar fluids. Prior attempts to rationalize the gelling power of a LMWG have been proposed based on various solubility indicators (dielectric constant, ${ }^{12-14} \mathrm{E}_{\mathrm{T}}$ polarity scale, ${ }^{14}$ Hildebrandt solubility parameter, ${ }^{15}$ Kamlet-Taft 40 parameters, ${ }^{14,16}$ Hansen solubility parameters ${ }^{14,17}$ ). Up to now, each of these attempts was focused on a particular LMWG family, and while the conclusions are of interest, they cannot be extended to other LMWGs. We presently report a general solubility parameter approach to predict the gelling ability of 45 any compound based on its behaviour in a limited set of solvents.

After dissolution of a LMWG in a solvent at elevated temperature and subsequent cooling, favourable and unfavourable interactions (i.e. solubility and insolubility) 50 must be balanced if a gel is to form instead of a solution or a precipitate. ${ }^{18-21}$ For example, it has been demonstrated in the case of a hydrogen bonded dendritic LMWG, that the gel-sol transition temperature is correlated to the polar and hydrogen bonding Hansen parameters. ${ }^{14}$ In a favourable domain, the
${ }_{55}$ solvent does not compete too much with inter-gelator hydrogen bonds, but still allows dissolution at high temperature. This important conclusion is however not directly applicable to other LMWG classes, in particular those involving no hydrogen bonds. Therefore, in order to derive a ${ }_{60}$ general guiding principle, one needs to consider the solubility parameter for the gelator as well as for the solvent.

In the field of polymer science, Hansen solubility parameters (HSPs) have been successfully used for several decades to select solvents for coating materials and other ${ }_{65}$ applications. $^{22}$ In this approach, the cohesive energy density is decomposed according to three contributions: dispersive interactions $\left(\delta_{d}\right)$, polar interactions $\left(\delta_{p}\right)$ and hydrogen bonds $\left(\delta_{h}\right)$. In practice, the procedure consists in comparing the HSPs for the solvent $\left(\delta_{d}^{s}, \delta_{p}^{s}, \delta_{h}^{s}\right)$ to the HSPs for the polymer $70\left(\delta_{d}, \delta_{p}, \delta_{h}\right)$. The distance between the two points in Hansen space is defined as $R=\sqrt{4\left(\delta_{d}-\delta_{d}^{s}\right)^{2}+\left(\delta_{p}-\delta_{p}^{s}\right)^{2}+\left(\delta_{h}-\delta_{h}^{s}\right)^{2}}$. If this distance is smaller than an empirical and case specific value $R_{\text {Sol }}$, then the polymer and the solvent have a high probability of being miscible. The values for the HSPs of 75 solvents can be found in the literature, ${ }^{23}$ and the values for the polymer are determined by testing the solubility of the polymer in a range of solvents and plotting the results in a $3 \mathrm{D}$ diagram, with $\delta_{d}^{s}, \delta_{p}^{s}$ and $\delta_{h}^{s}$ as axes. The points for the good solvents tend to cluster in a particular region in space, thus 80 defining a solubility sphere for the polymer, with $\left(\delta_{d}, \delta_{p}, \delta_{h}\right)$ as its centre and $R_{S o l}$ as its radius. ${ }^{24}$

We have tested the applicability of this procedure to the case of LMWGs by selecting from the literature 8 references that provide an extensive set of solubility data. ${ }^{25-32}$ The 85 structures of the selected gelators vary widely (Scheme 1) and involve the main supramolecular interactions to various extents: mainly hydrogen bonds (1-4) or ionic interactions $(5,6)$, or only $\pi$-staking, dipolar and van der Waals interactions $\mathbf{( 7 , 8 )}$. In each case, the solubility data reported in 90 the original article was analyzed and the solvents were grouped into three classes: S (solution forming), I (either insoluble or formation of a precipitate after cooling) and $G$ (gel formation). The results are plotted in Fig. 1 for gelator 1 and in $\mathrm{ESI}^{\dagger}$ for the others. 


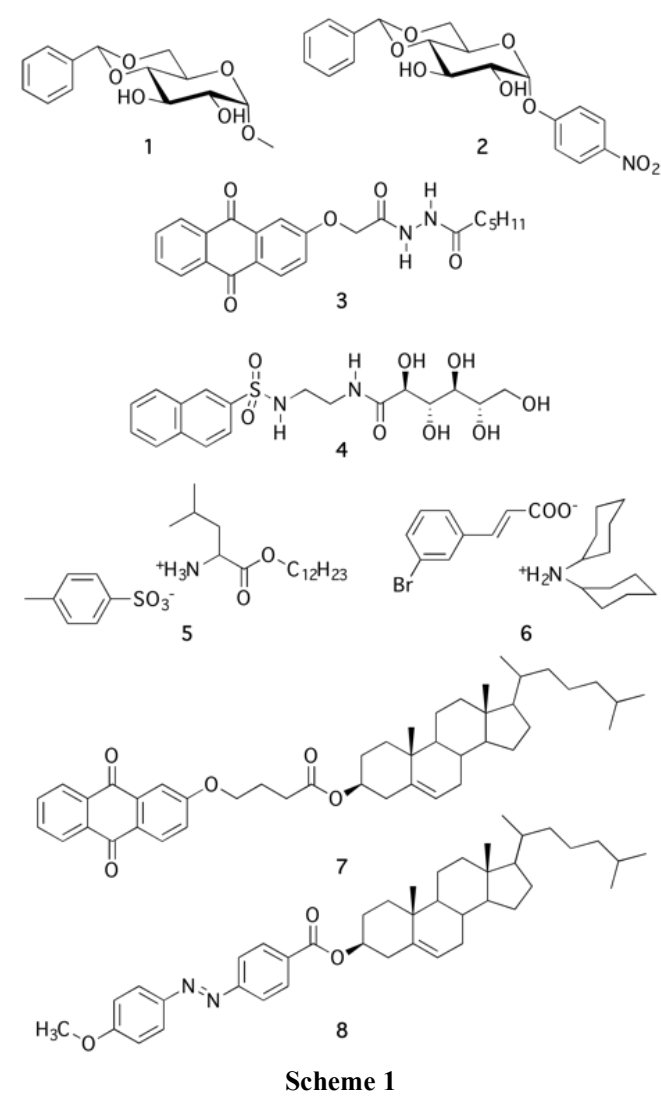

Fig. 1 qualitatively shows that there is a strong correlation between the solvent classes and the HSP: the good solvents ${ }_{5}(\mathrm{~S})$ are in a region of space with large values of $\delta_{p}^{S}$ and $\delta_{h}^{S}$; whereas the gelating solvents $(G)$ are clustered in a region of lower $\delta_{p}^{s}$ and $\delta_{h}^{s}$, but high $\delta_{d}^{s}$. This makes sense, because molecular dissolution requires a solvent that can efficiently compete with the main inter-gelator interaction (hydrogen 10 bonding in the case of $\mathbf{1}$ ), whereas gelation relies on the establishment of this interaction. The same qualitative pattern can be recognized with gelators $2,3,5$ and $\mathbf{6}$ which all contain strong hydrogen bond acceptors and donors (see ESI). Moreover, gelators 7 and $\mathbf{8}$, which do not contain such polar 15 groups, display an opposite behaviour: the good solvents (S) are in a region of space with low values of $\delta_{p}^{s}$ and $\delta_{h}^{s}$; whereas the gelating solvents $(\mathrm{G})$ are in a region of higher $\delta_{p}^{s}$ and $\delta_{h}^{s}$. The case of LMWG 4 is more complex: the hydrogen bonding solubility parameter $\left(\delta_{h}^{S}\right)$ for the gelating solvents

${ }_{20}(\mathrm{G})$ spans a large range of values (from 6 to $42 \mathrm{MPa}^{0.5}$ ). This feature is in agreement with the fact that $\mathbf{4}$ has been described as an ambidextrous gelator, i.e. a gelator of both water and organic solvents, ${ }^{31}$ and reveals the similar energetic weight of the hydrogen bonding groups and the non-polar naphthalene 25 group in the gel formation.

Remarkably, for all examples tested (including LMWG 4) the graphical representation of the HSPs shows a clear clustering of the solution forming solvents (S) in a region of space and of the gel forming solvents $(G)$ in a distinct region 30 of space. The fact that the $\mathrm{S}$ solvents are clustered together is not surprising, because we are probing the competition between solute-solvent and solute-solute interactions in the case of a molecularly dispersed solute. This is exactly the same situation as for polymer solubility studies, which have 35 been shown to be adequately described by the HSP approach. ${ }^{22}$ The fact that the $G$ solvents are not randomly mixed with the I solvents, but also form clusters of their own is a new and unexpected result. ${ }^{\star}$ The gelation domain defined by these points $(G)$ informs us on the interactions necessary to 40 stabilize the fibres and is probably related to their surface composition.

Fig. 1 Solubility data for LMWG $\mathbf{1}^{29}(30 \mathrm{~g} / \mathrm{L})$ represented in Hansen

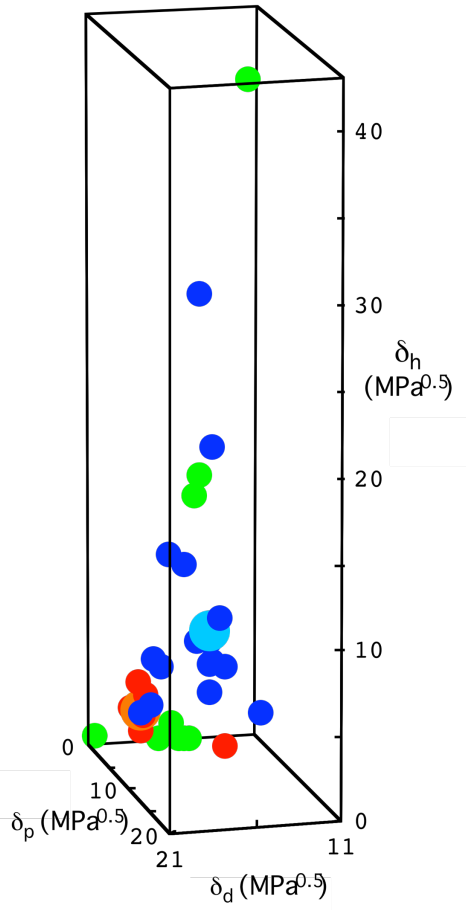

space. Blue: Soluble; red: Gel; green: Insoluble. Cyan: centre of the 45 solubility sphere; orange: centre of the gelation sphere.

In order to turn this experimental observation into a predictive tool, a quantitative description is needed. First, the centre and the radius of the solubility sphere were determined such that as many $\mathrm{S}$ solvents as possible lie inside the sphere, ${ }_{50}$ but I and G solvents lie outside (see ESI). ${ }^{22,24}$ Similarly, the centre and the radius of the gelation sphere were determined so that $\mathrm{G}$ solvents lie inside the sphere, but I and S solvents lie outside. Then the distance between each solvent and the centre of each sphere was computed and plotted in Fig. 2. The 55 correlation is excellent: most $\mathrm{S}$ solvents are included in the solubility sphere while nearly all other solvents are excluded (Fig. 2a). Moreover, most $G$ solvents are included in the gelation sphere while all other solvents are excluded (Fig. 2b). The situation is very similar for gelators $3,4,5,6$ and 8 (see ${ }_{60}$ ESI). The fact that a few outlying solvents are present reminds us that the correlation cannot be expected to be perfect, as is well known for the prediction of polymer solubility. The description of the data by a gelation sphere in the case of gelators $\mathbf{2}$ and $\mathbf{7}$ is less satisfactory, but can be improved by ${ }_{65}$ considering two gelation spheres (see ESI). The fact that more 
than one gelation sphere may be necessary to describe the data is in fact quite natural, because it is known that the functional groups of the gelator that are exposed at the fibre surface depend on the solvent in contact with the fibre. ${ }^{31}$
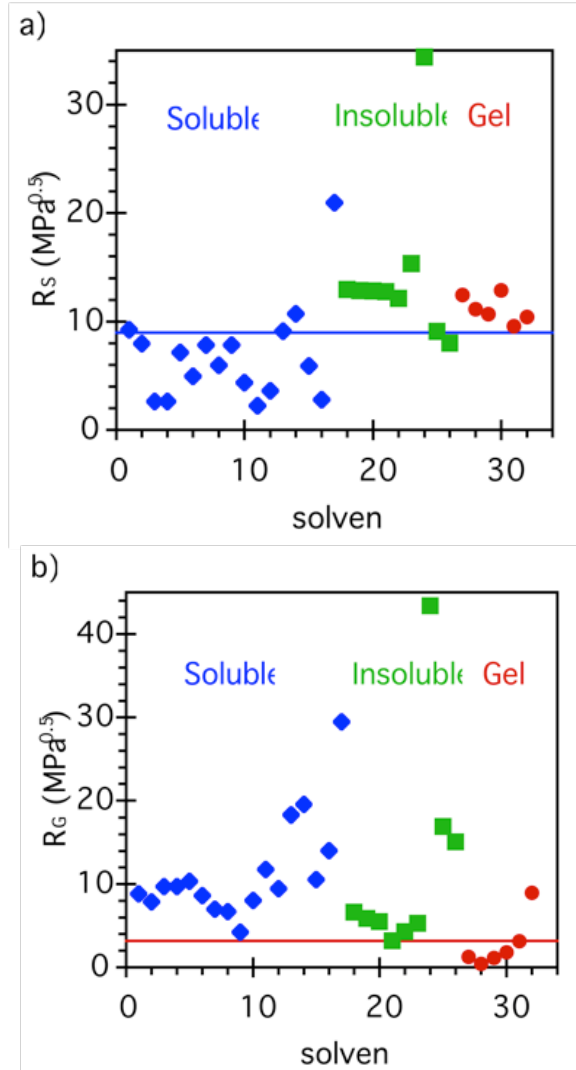

5

Fig. 2 Distances in Hansen space to the centre of the solubility sphere $\left[\delta_{d}\right.$ $\left.=16.0 ; \delta_{p}=9.4 ; \delta_{h}=8.7 ; R_{S o l}=9.0 \mathrm{MPa}^{0.5}\right]$ (a) or to the centre of the gelation sphere $\left[\delta_{d}=18.0 ; \delta_{p}=1.0 ; \delta_{h}=2.0 ; R_{G e l}=3.2 \mathrm{MPa}^{0.5}\right]$ (b) for

In conclusion, we propose a method to predict the behaviour of a known LMWG in untested solvents. Based on the behaviour of the LMWG in a set of solvents, it is possible to define a solubility sphere and one (or more) gelation 15 spheres. If the untested solvent HSPs fall in the solubility sphere or in the gelation sphere, then this solvent is likely to dissolve or to be gelled by the LMWG, respectively. Of course, the reliability of the prediction will depend on the quality of the initial solubility data set. $\$$ This straightforward 20 method should become a useful data mining tool, allowing to considerably reduce the number of trials usually involved during the identification of a suitable gelator for a particular application, i.e. in a particular formulation.

\section{Notes and references}

$25{ }^{a}$ UPMC Univ Paris 06, UMR 7610, Chimie des Polymères, F-75005

Paris, France.E-mail: laurent.bouteiller@upmc.fr

${ }^{b}$ CNRS, UMR 7610, Chimie des Polymères, F-75005 Paris, France

$\dagger$ Electronic Supplementary Information (ESI) available: Data treatment procedure and supporting figures. See DOI: $10.1039 / \mathrm{b} 000000 \mathrm{x} /$

30 \$ The metastable nature of organogels highlights the importance of kinetic effects, which are not included in the HSP approach. However, the present data shows that as long as the preparation conditions are consistent within each study (i.e. concentration and cooling rate), the thermodynamic HSP description is adequate.

$35 \$ \quad$ Typically, 5 to 10 solvents evenly distributed in Hansen space, within each class $\mathrm{S}, \mathrm{G}$, and I is enough to have a reliable estimation.

1 Molecular Gels: Materials with Self-Assembled Fibrillar Networks, ed. P. Terech and R. G. Weiss, Springer, Dordrecht, 2006.

2 M. George and R. G. Weiss, Acc. Chem. Res., 2006, 39, 489.

403 J. C. Stendhal, E. R. Zubarev, M. S. Arnold, M. C. Hersam, H.-J. Sue and S. I. Stupp, Adv. Funct. Mater. 2005, 15, 487.

4 B. Isare, L. Petit, E. Bugnet, R. Vincent, L. Lapalu, P. Sautet and L. Bouteiller, Langmuir, 2009, 25, 8400.

5 F. Fages, F. Vogtle and M. Zinic, Top. Curr. Chem., 2005, 256, 777.

456 N. M. Sangeetha and U. Maitra, Chem. Soc. Rev., 2005, 34, 821.

7 A. R. Hirst, B. Escuder, J. F. Miravet and D. K. Smith, Angew. Chem., Int. Ed., 2008, 47, 8002.

8 M. Llusar and C. Sanchez, Chem. Mater., 2008, 20, 782.

9 L. Frkanec and M. Zinic, Chem. Commun., 2010, 46, 522.

5010 A. Dawn, T. Shiraki, S. Haraguchi, S. Tamaru and S. Shinkai, Chem. Asian J., 2011, 6, 266.

11 J. W. Steed, Chem. Commun., 2011, 47, 1379.

12 A. Aggeli, M. Bell, N. Boden, J. N. Keen, P. F. Knowles, T. C. B. McLeish, M. Pitkeathly and S. E. Radford, Nature, 1997, 386, 259.

5513 J. Makarevic, M. Jokic, B. Peric, V. Tomisic, B. Kojic-Prodic and M. Zinic, Chem. Eur. J., 2001, 7, 3328.

14 A. R. Hirst and D. K. Smith, Langmuir, 2004, 20, 10851.

15 W. Frässdorf, M. Fahrländer, K. Fuchs and C. Friedrich, J. Rheol., 2003, 47, 1445.

6016 W. Edwards, C. A. Lagadec and D. K. Smith, Soft Matter, 2011, 7, 110.

17 K. Hanabusa, M. Matsumoto, M. Kimura, A. Kakehi and H. Shirai, $J$. Colloid Interface Sci., 2000, 224, 231.

18 A. R. Hirst, I. A. Coates, T. R. Boucheteau, J. F. Miravet, B. Escuder,

65 V. Castelletto, I. W. Hamley and D. K. Smith, J. Am. Chem. Soc., 2008, 130, 9113

19 J. H. van Esch, Langmuir, 2009, 25, 8392.

20 N. Zweep, A. Hopkinson, A. Meetsma, W. R. Browne, B. L. Feringa and J. H. van Esch, Langmuir, 2009, 25, 8802.

7021 J. Chen, J. W. Kampf and A. J. McNeil, Langmuir, 2010, 26, 13076.

22 C. M. Hansen, Hansen Solubility Parameters: A User's Handbook 2nd Ed.; CRC Press LLC: Boca Raton, FL, 2007; C. M. Hansen, Progress Org. Coat., 2004, 51, 77.

23 J. Brandrup, E.H. Immergut, E.A. Grulke, Polymer Handbook, fourth ed., Wiley, New York, 1999.

24 F. Gharagheizi, J. Appl. Polym. Sci., 2007, 103, 31.

25 K. Murata, M. Aoki, T. Suzuki, T. Harada, H. Kawabata, T. Komori, F. Ohseto, K. Ueda and S. Shinkai, J. Am. Chem. Soc., 1994, 116, 6664.

8026 R. Mukkamala and R. G. Weiss, Langmuir, 1996, 12, 1474.

27 N. Amanokura, K. Yoza, H. Shinmori, S. Shinkai and D. Reinhoudt, J. Chem. Soc., Perkin Trans. 2, 1998, 2585.

28 K. Hanabusa, H. Kobayashi, M. Suzuki, M. Kimura and H. Shirai, Colloid Polym. Sci., 1998, 276, 252.

8529 O. Gronwald and S. Shinkai, Chem. Eur. J., 2001, 7, 4329.

30 D. R. Trivedi, A. Ballabh, P. Dastidar and B. Ganguly, Chem. Eur. J., 2004, 10, 5311.

31 N. Yan, G. He, H. Zhang, L. Ding and Y. Fang, Langmuir, 2010, 26, 5909.

9032 J.-W. Liu, J.-T. Ma and C.-F. Chen, Tetrahedron, 2011, 67, 85. 